\title{
Repression of the gene encoding the TGF- $\beta$ type II receptor is a major target of the EWS-FLI1 oncoprotein
}

Ki-Baik Hahm et al.

Nature Genet. 23, 222-227 (1999).

In the abstract, "ES cells" were indicated to be embryonic stem cells, when in fact they were Ewing sarcoma cells.

\section{Delayed early embryonic lethality following disruption of the murine cyclin A2 gene}

Martin Murphy et al.

Nature Genet. 15, 83-86 (1997).

Maternally derived cyclin A2 was suggested to ensure normal cell division cycles in $C c n a 2^{-/}$mouse embryos. This hypothesis was based on immunofluorescence studies at the blastocyst stage. The one-step fixation and permeabilization procedure followed by incubation with the anti-cyclin A2 antiserum has since been shown to result in a high background staining of the embryo. Given that maternal cyclin A2 is undetectable in $\mathrm{Ccna2}^{-/-}$blastocysts using other fixation and permeabilization procedures, we feel that maternal cyclin A2 cannot be considered to persist to the blastocyst stage during early mouse development. This point does not change any of the other conclusions presented in the article. 